\title{
A Complement-Based Fast Algorithm to Generate Universal Test Sets for Multi-Output Functions
}

\author{
Beyin Chen and Chung Len Lee, Senior Member, IEEE
}

\begin{abstract}
In this paper, a fast universal test set (UTS) generation algorithm for multi-output functions is presented.

The algorithm first generates the UTS for single-output functions by directly Shannon-expanding and complementing the function. This significantly reduces the time complexity and the usage of temporary memory. Also, it stores tests in test cubes to save the size of memory for test storing. Two-six orders of magnitude in computation efficiency improvement and 1-1800 fold for memory saving over the conventional method are achieved. It then merges the generated test cubes for each singleoutput function into a set of mutually disjoint test cubes to be the UTS for a multi-output function by employing a new compaction technique. The size of UTS thus obtained is 1-20 times smaller than that of UTS without compaction.
\end{abstract}

\section{INTRODUCTION}

B ASED ON THE unate function theory [1], a universal test set (UTS) for combinational circuits was proposed by Akers [2] and Reddy [3]. It was shown that the UTS can be generated from the functional description and can detect all single and multiple stuck-at faults of the circuit implementation which satisfies "unate gate network" [3] property for the function. Also, the UTS can be paired with a universal initialization set to detect every detectable stuck-open fault in a "restricted CMOS circuit" [4]. However, the procedure to generate the UTS involves a process to enumerate the truth table of the function and it has an exponential complexity Also, even the computation can be speeded up, the size of UTS grows exponentially with the number of binate input variables. This makes the storage of test patterns a problem. Moreover for a multi-output function, the compaction of compatible tests among the UTSs of the single-output functions is also a problem which has never been considered by researchers.

This paper first presents a fast algorithm, FUTS, to generate UTS for single-output functions. The algorithm adopts a method to find UTS by Shannon--expanding and complementing the function, hence completely eliminates the truth table enumeration process. Also, the method treats the test information in terms of "test cubes" instead of "test patterns," and this solves the storage problem for the UTS. Experimental results show that the algorithm achieves improvements of $10^{2}-10^{6}$ times in the computation efficiency and $1-1800$ times in the storage saving of test sets over the conventional method.

Manuscript received February 18, 1993. This work was supported by The National Science Council, Republic of China, under Grant NSC82-0404-E009183. This paper was recommended by Associate Editor K.-T. Cheng.

The authors are with the Department of Electronics Engineering \& Institute of Electronics, National Chiao Tung University, Hsinchu, Taiwan, Republic of China.

IEEE Log Number 9214057
Then this paper presents a compaction technique to merge the generated test cubes into a set of mutually disjoint test cubes to be the UTS for multi-output functions. Experimental results show that the size of UTS generated with the technique is 1-20 times smaller than that of UTS without compaction.

\section{DENOTATIONS}

In this section, some terms and denotations which are used in this paper are first given.

A logic function $F$ has $n$ input variables $x_{1}, x_{2}, \ldots, x_{n}$ and is represented in the sum-of-products form: $F=P_{1}+P_{2}+$ $\cdots+P_{k}$, where $P_{j}$ is represented by a cube $c_{j}$ [5].

The set of $k$ cubes defined as above is said to be a cover of $F$, denoted as $\operatorname{cover}(\mathbf{F})$. For the cover(F), it can be minimized to be prime and irredundant [5], which is denoted as mini_cover $(\mathbf{F})$.

The expanded truth table of a logic function is the truth table of the input literals of the input variables. In the expanded truth table, an input vertex $X$ dominates the input vertex $Y$ if and only if the entry of $X$ is 1 where the corresponding entry of $Y$ is 1 . For example, if $X=1101$ and $Y=1001$ then $X$ dominates $Y$. A minimal true vertex of a logic function is the input vertex that does not dominate any other true vertex except itself. A maximal false vertex of a logic function is the input vertex that is not dominated by any other false vertex except itself. The UTS of a logic function $F$ is the union of the minimal true vertex set and the maximal false vertex set of the expanded truth table of $F$ [2], [3]. It is denoted as: $\mathbf{U T S}(\mathbf{F})=\mathbf{V}_{\operatorname{mint}}(\mathbf{F})+\mathbf{V}_{\operatorname{maxf}}(\mathbf{F})$.

An example as shown in Fig. 1 is to demonstrate the above, where the sum-of-products form, cubical representations, and the expanded truth table of $F$ are shown in Fig. 1 (a), (b), and (c) respectively. The $V_{\operatorname{mint}}(F)$ and $V_{\operatorname{maxf}}(F)$ obtained, which constitute the UTS of $F$, are shown in Fig. 1 (d).

In the Shannon expansion of the function $F$, i.e., $F=$ $x_{i} \cdot F_{x_{i}}+\overline{x_{i}} \cdot F_{\overline{x_{i}}}, F_{x_{i}}$ and $F_{\overline{x_{i}}}$ are the one-cofactor and the zero-cofactor of $F$ with respect to the splitting variable $x_{i}$ respectively. A cofactor is strict_unate if it is independent of all the binate input variables of $F$. A cofactor is tautology or nil if it is always logic true or false respectively for all its input combinations. It is seen that tautology and nil are two special cases of strict_unate cofactors.

The UTS generated by the method of this paper is represented by test_cubes instead of the conventional test patterns. A test_cube is a subset of UTS represented by a cube. A test_cube is said to be true with respect to an output $F$ if it makes the output logic 1 . It is false if it makes the output logic 


$$
F=\bar{a} b \bar{c} \bar{d}+\bar{b} \bar{c} \bar{d}+\bar{a} c \bar{d}+\bar{a} \bar{b} c d
$$

(a)

$\operatorname{cover}(F)=\{[0101],[-000],[0-10],[0011]\}$. mini_cover $(F)=\{[0101],[-000],[0-10],[001-]\}$.

(b)

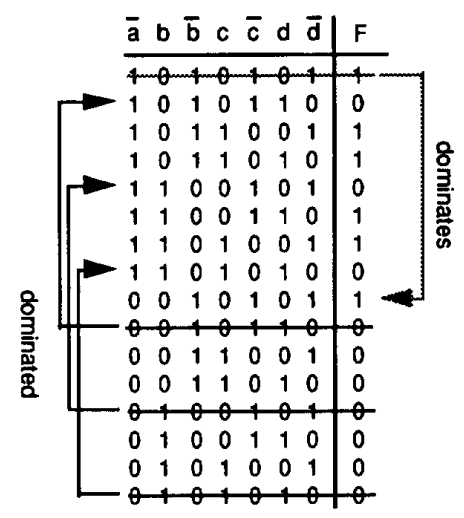

(c)

$V_{\operatorname{mint}}(F)=\{[a b c d]:[1000],[0010],[0110],[0101],[0011]\}$.

$V_{\operatorname{maxf}}(F)=\{[a b c d]:[0100],[1010],[1110],[0001],[1101],[1011],[0111])$.

(d)

Fig. 1. An example to demonstrate the conventional method to generate the universal test set. (a) Sum-of-products form of function $F$. (b) Cubical representation of $F$. (c) Expanded truth table of $F$. (d) The minimal true vertex set and maximal false vertex set of $F$.

0 . The test_cover of $F$, denoted as test_cover $(F)$, contains all the true and false test_cubes of $F$, and is the $\operatorname{UTS}(F)$. For example, the UTS $(F)$ in Fig. 1 contains 5 true tests and 7 false tests, which can be represented by 4 true test_cubes $=\{[1000],[001-],[0110],[0101]\}$ and 6 false test_cubes $=$ $\{[0100],[101-],[1110],[0001],[1101],[0111]\}$. Note that in the above, test_cube (001-) represents test patterns (0010) and (0011).

\section{A FAST ALgORITHM TO GENERATE UTS FOR SINGLE-OUTPUT FUNCTIONS}

To present the algorithm FUTS, a theorem and some propositions and lemmas are first presented.

Proposition 1: For an input variable $x_{i}$ of function $F$, if it is positively (negatively) unate for $F$, then it is negatively (positively) unate for $\bar{F}$, which is the complement of $F$. If $x_{i}$ is binate for $F$, then it is still binate for $\bar{F}$.

Applying Proposition 1 to the definition of $V_{\operatorname{maxf}}(F)$ and $V_{\text {mint }}(\bar{F})$, the following proposition holds.

Proposition 2: For a function $F$, its maximal false vertex set is equal to the minimal true vertex set of its complement $\bar{F}$, i.e., $V_{\operatorname{maxf}}(F)=V_{\operatorname{mint}}(\bar{F})$.

Theorem 1: $\operatorname{UTS}(F)=x_{i}\left[U T S\left(F_{x_{i}}\right)\right]+\overline{x_{i}}\left[U T S\left(F_{\overline{x_{i}}}\right)\right]$, where $x_{i}$ is a binate input variable of $F$.

Proof: For two row vectors $X$ and $Y$ in the expanded truth table of $F$, if there exists a binate input variable for which the values of $X$ and $Y$ are different, then $X$ and $Y$ never dominate each other. So, to find $\operatorname{UTS}(F)$, we can divide the expanded truth table into two for $x_{i}$ to be 0 and 1 and then find their UTS's respectively. Based on this and the definition of Shannon expansion, this theorem holds.

With Theorem 1, a binate function $F$ can be recursively expanded into two cofactor functions, and this recursion can be continued until all the cofactor functions are strict_unate. The UTS of $F$ can be obtained directly by using Theorem 1 by combining the UTS's of the cofactor functions.

For a cofactor function $C$ which is strict_unate, Lemmas 1 , 2 , and 3 can be used to obtain its test_cubes.

Lemma I: If $C$ is tautology, then UTS $(C)$ contains a single true test_cube $t$ and it can be obtained as follows:

for $(i:=I$ to $n)$

if ( $x_{i}$ is not split) then

$t^{i}=0$ if $x_{i}$ is positively unate w.r.t. $F$;

$t^{i}=1$ if $x_{i}$ is negatively unate w.r.t. $F$;

$t^{i}=-$ if $x_{i}$ is binate w.r.t. $F$,

where $t^{i}$ is an entry of the true test_cube $t=\left[t^{1}, \ldots, t^{n}\right]$.

Lemma 2: If $C$ is nil, then UTS $(C)$ contains a single false test_cube $f$ and it can be obtained as follows :

for $(i:=1$ to $n)$

if ( $x_{i}$ is not split) then

$f^{i}=1$ if $x_{i}$ is positively unate w.r.t. $F$;

$f^{i}=0$ if $x_{i}$ is negatively unate w.r.t. $F$;

$f^{i}=-$ if $x_{i}$ is binate w.r.t. $F$,

where $f^{i}$ is an entry of the false test_cube $f=\left[f^{1}, \cdots, f^{n}\right]$.

Lemma 3: If $C$ is strictunate and is neither tautology nor nil, and mini_cover $(C)$ and mini_cover $(\bar{C})$ have $k$ cubes $c_{1}, \ldots, c_{k}$ and $m$ cubes $b_{1}, \ldots, b_{m}$ respectively, then UTS $(C)$ contains $k$ true test_cubes $t_{1}, \ldots, t_{k}$ and $m$ false test_cubes $f_{1}, \cdots, f_{m}$ which can be obtained as follows :

for $(j:=l$ to $k)$

for $(i:=1$ to $n)$

if ( $x_{i}$ is not split) then

$t_{j}^{i}=c_{j}^{i}$ if $c_{j}^{i}=1$ or 0 ;

$t_{j}^{i}=0$ if $c_{j}^{i}=2$ and $x_{i}$ is positively unate w.r.t. $F$;

$t_{j}^{i}=1$ if $c_{j}^{i}=2$ and $x_{i}$ is negatively unate w.r.t. $F$;

$t_{j}^{i}=-$ if $c_{j}^{i}=2$ and $x_{i}$ is binate w.r.t. $F$,

where $t_{j}^{i}$ is an entry of the true test_cube $t_{j}=\left[t_{j}^{1}, \ldots, t_{j}^{n}\right]$; and

for $(j:=1$ to $m)$

for $(i:=l$ to $n)$

if $\left(x_{i}\right.$ is not split) then

$f_{j}^{i}=b_{j}^{i}$ if $b_{j}^{i}=1$ or 0 ;

$f_{j}^{i}=1$ if $b_{j}^{i}=2$ and $x_{i}$ is positively unate w.r.t. $F$;

$f_{j}^{i}=0$ if $b_{j}^{i}=2$ and $x_{i}$ is negatively unate w.r.t. $F$;

$f_{j}^{i}=-$ if $b_{j}^{i}=2$ and $x_{i}$ is binate w.r.t. $F$,

where $f_{j}^{i}$ is an entry of the false test_cube $f_{j}=\left[f_{j}^{1}, \cdots, f_{j}^{n}\right]$.

The proofs of Lemmas 1,2 , and 3 can be done by applying Propositions 1 and 2 and Theorem 1.

For the example function of Fig. 1, the Shannon expansion process for $F$ to find its test_cubes can be represented by a binary tree as shown in Fig. 2(a), where $T$ and $N$ are cofactors which are tautology and nil respectively. After applying Lemmas 1,2 , and 3 to the co factors and merging the test_cubes by setting the splitting variables to either ' 0 ' or ' 1 ' according to the path values in the binary tree, the 


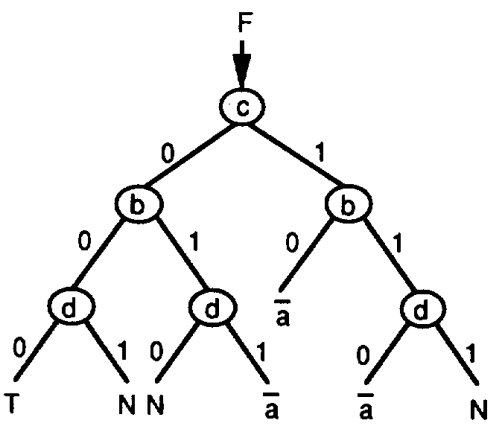

(a)

\begin{tabular}{|c|c|}
\hline \multicolumn{2}{|c|}{ test_cover(F) } \\
\hline abcd & $F$ \\
1000 & 1 \\
0001 & 0 \\
0100 & 0 \\
0101 & 1 \\
1101 & 0 \\
$001-$ & 1 \\
$101-$ & 0 \\
0110 & 1 \\
1110 & 0 \\
0111 & 0 \\
\hline
\end{tabular}

(b)
Fig. 2. The demonstration of the algorithm FUTS using the example of Fig. 1. (a) Shannon expansion process for $F$ to find its test_cubes. (b) The test_cover of $F$ which constitutes the UTS of $F$.

test_cubes of $F$ are generated and shown in Fig. 2(b) which is the test_cover of $F$.

As demonstrated with this example, the expanded truth table enumeration and the comparing operations are completely eliminated. Also, for the conventional method, 12 tests as shown in Fig. 1 were generated, while here, only 10 test_cubes are generated. In general, the size of memory for test storing is largely reduced by using this method.

Finally, it is specially mentioned that to generate the test_cover of an output function using FUTS, the intersection of any two test_cubes in the test_cover is empty.

The details of the algorithm FUTS are presented as follows: Algorithm FUTS(F);

INPUT: $\mathrm{F} / *$ a set of cubes. $* /$

OUTPUT: test_cubes ${ }^{*}$ a set of test_cubes that constitutes the UTS of $F . * /$

$$
\{
$$

Make $F$ a prime cover.

Scan the prime cover, check for every input variable to determine whether it is positively, negatively unate, or binate.

test_cubes := UTS_GEN $(F)$;

\}

Procedure UTS_GEN(cofactor);

\{

if $($ cofactor $==$ nil) then

$/^{*}$ Lemma 2 is applied. */ test_cubes := NIL_CASE( );

else if (there is a row of all '-'s in cover(cofactor))

$f^{*}$ Lemma 1 is applied. */ test_cubes := TAUTOLOGY_CASE( );

else if (cofactor is strict_unate)

$f^{*}$ Lemma 3 is applied. *f test_cubes := UNATE_CASE(cofactor);

else

$l^{*}$ Theorem 1 is applied. */ $x_{j}:=$ SPLIT_SELECT(cofactor);

$f^{*}$ In general, a most binate variable of cover(cofactor) is selected. */

\author{
one-cofactor : $=$ ONE_COF(cofactor, $x_{j}$ ); \\ zero-cofactor := ZERO_COF(cofactor, $x_{j}$ ); \\ $1 *$ Compute the one-cofactor and the zero-cofactor \\ w.r.t. $x_{j}$ respectively. $*$ / \\ test_cubes := MERGE (UTS_GEN(one-cofactor), \\ UTS_GEN(zero-cofactor), $x_{j}$ ); \\ ${ }^{*}$ The variable $x_{j}$ in test_cubes generated from \\ UTS_GEN(one-cofactor) is set to $1 . *$ / \\ $f^{*}$ The variable $x_{j}$ in test_cubes generated from \\ UTS_GEN(zero-cofactor) is set to $0 . * /$ \\ return (test_cubes); \\ \} \\ Procedure UNATE_CASE(cofactor); \\ \{ \\ mini_cover := UNATE_SIMPLIFY (cofactor); \\ Generate $k$ true test_cubes corresponding to the $k$ cubes \\ in mini_cover; $/ *$ Lemma $3 *$ / \\ comp_ cover := UNATE_COMPLEMENT (mini_ cover); \\ Generate $m$ false test_cubes corresponding to the $m$ cubes \\ in comp_cover; $/ *$ Lemma $3 * /$ \\ return (the $k$ true test_cubes + the $m$ false test_cubes); \\ \} \\ Note that in the above Procedure UNATE_CASE( ), \\ UNATE_SIMPLIFY( ) is to make a unate cover prime and \\ irredundant and UNATE_COMPLEMENT( ) is to compute the \\ complement of a unate cover. There are published procedures \\ [5] which are simple and fast to do the above. In addition, if \\ UNATE_COMPLEMENT( ) is used to complement a prime \\ and irredundant cover, e.g., mini_cover $(C)$, the returned cover, \\ $\overline{\mathrm{C}}$, is prime and irredundant too. So, comp_cover is prime and \\ irredundant and Lemma 3 can be applied.
}

\section{UTS COMPACTION FOR MULTI-OUTPUT FUNCTIONS}

To generate tests from a multi-output function, all the outputs of the circuit of the realized function are assumed to be independent of one another and every output circuit satisfies the property of "unate gate network" as proposed in [3]. The multi-output UTS can be easily obtained by finding the UTS for every single-output function by using the UTS generation algorithm FUTS and then combining the UTS for every singleoutput function. However, since the test_covers generated for the single-output functions have compatible test patterns of one another, it needs to compact the tests among test_covers. Since the number of patterns of the generated UTS may increase exponentially to the number of the function inputs, it is impossible to compact them in a reasonable time if the conventional compaction method, which can only compact test patterns, is used. In the following, some denotations are given and then a procedure is proposed to compact tests directly from test_cubes.

For a multi-output function, any of its single-output is usually dependent on a subset of primary inputs. The inputs that do not belong to an output function are independent inputs (or don't care inputs) with respect to the output function and are represented by $X$ (don't care) in the generated test_cubes. For example, consider a function which has two outputs $F$ and $G$. Assume that their test_cubes are as 


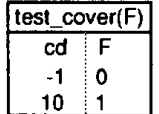

\begin{tabular}{|c:c|}
\hline test_cover(G) \\
\hline abd & $\mathrm{G}$ \\
101 & 1 \\
011 & 0 \\
\hline
\end{tabular}

(a)

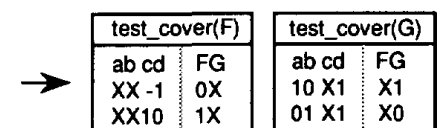

(b)
Fig. 3. An example to demonstrate the test_cover representation for multi-output functions.
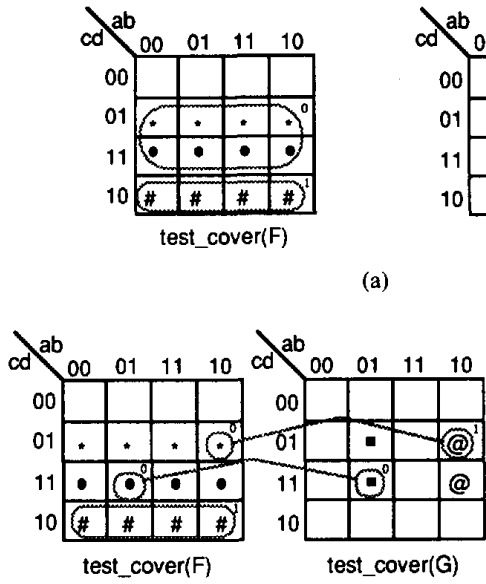

(b)

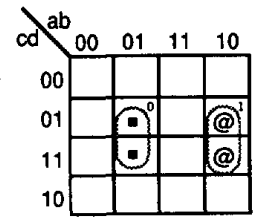

test_cover(G)

\begin{tabular}{|c|c|}
\hline \multicolumn{2}{|c|}{ test_cover(F,G) } \\
\hline$a b c d$ & FG \\
\hline 1001 & 01 \\
\hline 0111 & 00 \\
\hline$x \times 10$ & $1 x$ \\
\hline
\end{tabular}

(c)

Fig. 4. (a) Karnaugh map representation for test_covers of $F$ and $G$ of Fig. 3(b). (b) An optimum compaction performed from the Karnaugh map of (a). (c) The cubical representation of the compaction result of (b).

shown in Fig. 3(a). Since $F$ and $G$ are independent of $\{a, b\}$ and $c$ respectively, test_cover $(F)$ and test_cover $(G)$ can be reexpressed as shown in Fig. 3(b). The columns correspond to inputs $a, b$ in test_cover $(F)$ and input $c$ in test_cover $(G)$ are all $X$ 's. Note that the meaning of the symbol '-' is the same as mentioned in the previous sections. For example, the false test_cube ( $X X-1)$ of $F$ as shown in Fig. 3(b) contains two test patterns $(X X 01)$ and $(X X 11)$.

The example of Fig. 3 is used to demonstrate the problem to compact tests between two test_covers. Fig. 4(a) shows the Karnaugh map representation of the test_covers of $F$ and $G$ of Fig. 3(b), where each type of symbols of the entries of the maps represents a test. For example, test_cover(F) contains three tests, so there are three types of symbols. Also since there are two independent inputs, i.e., $a$ and $b$, for output function $F$, there are four choices for $a, b$ for the three tests respectively. The problem to find the test_cover $(F, G)$ is to find a minimal set of input patterns to cover all types of symbols in the Karnaugh maps. In this example, there exist two optimum solutions for test_cover $F, G)$, one of which is shown in Fig. 4(b) and its cubical representation is shown in Fig. 4(c). It is noted that don't cares ( $X$ 's) are preserved for further compaction if there are more outputs.

Since the test compaction is a problem equivalent to the minimum coloring problem, which is NP-complete, in the following, two operations for test_cubes are defined and a heuristic compaction method is proposed to solve the problem.

It is the order that the test_covers are to be compacted affects the compaction efficiency. A weight: Essentiality(TC) for a

\begin{tabular}{c|cccc}
$\cap$ & 0 & 1 & $x$ & - \\
\hline 0 & 0 & $\Psi$ & 0 & 0 \\
1 & $\Psi$ & 1 & 1 & 1 \\
$x$ & 0 & 1 & $x$ & 0 \\
- & 0 & 1 & 0 & -
\end{tabular}

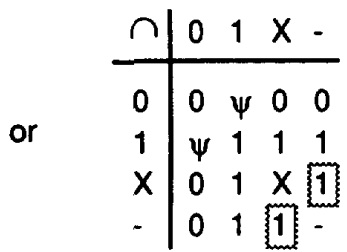

Fig. 5. The operation rules for test_cube intersection.

test_cover TC, is first defined to guide the ordering to compact the test_covers.

$$
\text { Essentiality(TC) }=1-\frac{\text { The number of don't care inputs of TC }}{\text { The number of total inputs }}
$$

The value of an essentiality is 1 if the test_cover is a function of all inputs, and is 0 if the test_cover is independent of all the inputs. The larger of the essentiality of the test_cover is, the earlier of the test_cover should be selected to be compacted.

A procedure, TEPACT, is developed to compact test_covers. TEPACT selects two test_covers at one time, according to the weights of the test covers, to do compaction. To do compaction, it invokes a cover compaction operation to compact every test_cube of a test_cover with the test cubes of the other test_cover in a one by one order. The cube compaction operation involves two steps:

Step 1. Perform the intersection operation to obtain a compatible cube of the two cubes.

Step 2. Delete the compatible cube from the two cubes respectively.

The intersection operation ( $\cap$ ) of two cubes is to find their compatible cube. The operation rules are summarized in Fig. 5. A $\psi$ generated during the intersection process for two cubes means that the two cubes are not compatible and no compatible cube is generated. In the table, it is noted that $X \cap$ - is defined to be either 0 or 1 .

To do the operation of Step 2, the disjoint sharp operation (*) which was defined in [6] is used. The operation can be used to delete the compatible cube of a cube from itself and make the resultant cubes mutually disjoint. It may generate more than one cubes. For example: $(X--0) *(1000)=$ $\{(X 1-0),(X 010)\}$.

The procedure of TEPACT is:

Procedure TEPACT(test_covers)

/* INPUT: the test_covers generated by FUTS. OUTPUT : test_cover_H $H *$ /

Step 1. Compute Essentiality for every test_cover. Determine the order of the test_covers to be compacted according the Essentiality value;

Step 2. Get two test_covers, test_cover_F and test_cover_G;

Step 3. Perform cover compaction : test_cover_H $H=$ cover_compaction(test_cover_ $F$,test_cover_ $G$ );

Step 4. If there is test_cover uncompacted, then let test_cover_F $F$ $:=$ test_cover_$H$ and test_cover_$G:=$ next test_cover, go to Step 3.

Otherwise, return test_cover $H$ and exit from this procedure. We use the example of Fig. 3 to demonstrate TEPACT. 


\begin{tabular}{|c|c|c|c|c|c|}
\hline \multicolumn{4}{|c|}{ lest_cover(G) lest_cover(F) } & \multirow{2}{*}{$\begin{array}{c}\begin{array}{c}\text { compatible } \\
\text { test_cover(F,G) }\end{array} \\
\varnothing\end{array}$} & \multirow{2}{*}{$\begin{array}{l}\text { Comments } \\
\text { Initial State. }\end{array}$} \\
\hline $\begin{array}{l}10 \times 1 \\
01 \times 1\end{array}$ & $\begin{array}{l}x_{1} \rightarrow \\
x_{0}\end{array}$ & $\begin{array}{l}X X-1 \\
X X 10\end{array}$ & $\begin{array}{l}0 x \\
1 x\end{array}$ & & \\
\hline $01 \times 1$ & X0₹承 & $\begin{array}{l}X X 10 \\
X X 11\end{array}$ & $\begin{array}{l}1 X \\
0 x\end{array}$ & 100101 & $\begin{array}{l}\text { Result of the first time } \\
\text { of cube compaction. }\end{array}$ \\
\hline$\ell$ & & XX10 & $1 x$ & $\begin{array}{ll}1001 & 01 \\
0111 & 00\end{array}$ & $\begin{array}{l}\text { Result of the second time } \\
\text { of cube compaction. }\end{array}$ \\
\hline
\end{tabular}

(a)

test_cover_H $=$ compatible test_cover $(F, G)+$ test_cover $(G)+$ test_cover $(F)$ $=\left\{\left(\begin{array}{ll}1001 & 01\end{array}\right),\left(\begin{array}{lll}0111 & 00\end{array}\right),(X X 101 X)\right\}$

(b)

Fig. 6. The demonstration of the process of TEPACT using the example of Fig. 3. (a) Test_cover compaction process. (b) The resultant test_cover after the compaction process of (a).

First, since the essentiality of $G$ is larger, the test_cover $(G)$ is selected to compact with the test_cover $(F)$. Initially, an empty cover, compatible test_cover $(\mathbf{F}, \mathbf{G})$ is created to store the compatible cubes of test_covers $F$ and $G$, as shown in the first row of Fig. $6(\mathrm{a})$. The cube $(10 X 1, X 1)$ is intersected with $(X X-1,0 X)$ and the resultant cube $(1001,01)$ is put into compatible test_cover $(F, G)$. $(1001,01)$ is then disjoint sharped with $(10 X 1, X 1)$ and $(X X-1,0 X)$ respectively. The results are $\emptyset$ (empty set) and $(X X 11,0 X)$ respectively. The old cubes $(10 X 1, X 1)$ and $(X X-1,0 X)$ are then removed and the new cube $(X X 11,0 X)$ is appended to test_cover $(F)$. This result of cube compaction is shown in the second row of Fig. 6(a). The same process is repeatedly performed until one of the two test_covers is empty. The resultant test_cover_ $H$ after this compaction process is shown in Fig. 6(b). It is seen that the number of tests is reduced from five to three and this is the optimum compaction result which is shown in Fig. 4(c).

With the above, the procedure, MOUTS, to generate the UTS for a multi-output function is summarized as follows:

Procedure MOUTS /* To generate the UTS for a multi-output function */

Step 1. Extract all the single-output functions from the original multi-output function. Find their independent input sets respectively.

Step 2. Generate the test_cover for every single-output function using the UTS generation algorithm FUTS.

Step 3. Perform procedure TEPACT for the generated test_covers to obtain the UTS of the multi-output function.

\section{EXPERIMENTAL RESULTS}

\section{A. Results on FUTS}

The above algorithm and the conventional method to find UTS have been implemented in $C$ language to run on a sun4/SPARC2 workstation. They are denoted as FUTS and CUTS respectively and were applied to run on 18 benchmark functions [5], which are represented as sum-of-products forms, to generate UTS. Since these benchmark functions are multiple-output functions, for each of them, we randomly select and extract an output as a single-output function. Table
TABLE I

The Run Results on 18 Benchmark Functions [5] By FUTS and CUTS

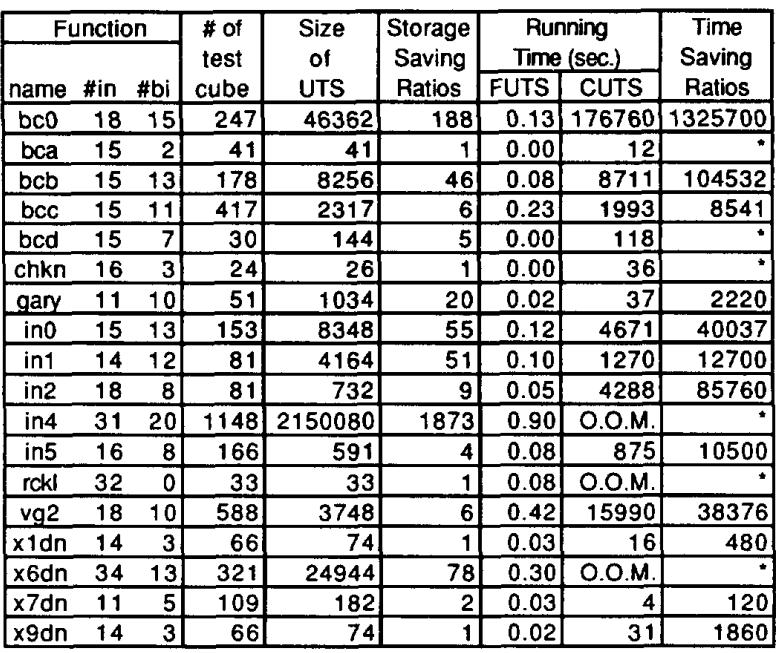

Storage Saving Ratios : test-storing saving ratios.

O.O.M. : out of memory. " : the ratio can not be calculated.

I gives the results obtained for FUTS and CUTS. In the table, column 2 is the number of input variables, column 3 is the number of binate input variables. Column 4 shows the number of test_cubes obtained with FUTS, column 5 shows the size of UTS, and column 6 , which is obtained by dividing column 5 by column 4 , is the memory saving of using "test_cubes" to store the test patterns. Column 7 and column 8 are the CPU times spent by FUTS and CUTS to obtain UTS respectively, and column 9 is the ratio of improvement of FUTS over CUTS obtained by dividing column 8 by column 7 . It can be seen that $10^{2}-10^{6}$ fold improvement is obtained for these 18 functions. It is to be specially mentioned that, for the functions: rckl, $\mathrm{x} 6 \mathrm{dn}$, in 4 , whose input numbers are larger than 30 , more than $2^{30}$ bytes ( $=1000$ Mbytes) of temporary memory are needed for CUTS for truth table enumeration! Yet, for FUTS, for the largest benchmark function, in 4 , only $1 \mathrm{~s}$ of CPU time was spent to obtain its UTS and $4 \mathrm{~K}$-byte memory was used to store its test_cubes.

To demonstrate the efficiency of the generated UTS, the functions in Table I are synthesized by the multi-level logic synthesizer misII [7], and then the fault coverages for these circuits are simulated for both the generated UTS's in Table I and the same number of randomly generated patterns. The simulation results are shown in Table II. In the table, column 2 is the number of gates synthesized by misII. Column 3 is the number of detectable faults (the number in parenthesis is the number of redundant faults.). Column 4 is the number of simulated patterns. Column 5 and column 6 show the fault coverages obtained by simulating the UTS's and the randomly generated patterns respectively. The fault coverages in both the columns are obtained by dividing the number of detected faults by the number of detectable faults. We can see that the generated UTS's reach $100 \%$ fault coverages for all the circuits. But for random patterns, the fault coverages may be low to $3 \%$. 
TABLE II

The Fault Simulation Results for the Generated UTS'S in TABLE I AND THE SAME Number OF RANDOM PaTterns

\begin{tabular}{|r|r|r|r|r|r|}
\hline & & & & \multicolumn{2}{|c|}{ Fault Coverages } \\
\cline { 5 - 6 } Fun. & \#gate & \#rault & \#pattern & \multicolumn{1}{c|}{ UTS } & random \\
\hline bc0 & 120 & 600 & 46362 & $100.00 \%$ & $98.00 \%$ \\
\hline bca & 15 & 68 & 41 & $100.00 \%$ & $4.41 \%$ \\
\hline bcb & 65 & 346 & 8256 & $100.00 \%$ & $79.48 \%$ \\
\hline bcc & 175 & 906 & 2317 & $100.00 \%$ & $62.47 \%$ \\
\hline bcd & 17 & 92 & 144 & $100.00 \%$ & $3.26 \%$ \\
\hline chkn & 15 & 78 & 26 & $100.00 \%$ & $24.36 \%$ \\
\hline gary & 30 & $(+2) 152$ & 1034 & $100.00 \%$ & $100.00 \%$ \\
\hline in0 & 77 & $(+4) 384$ & 8348 & $100.00 \%$ & $98.63 \%$ \\
\hline in1 & 60 & $(+1) 283$ & 4164 & $100.00 \%$ & $100.00 \%$ \\
\hline in2 & 27 & 138 & 732 & $100.00 \%$ & $78.26 \%$ \\
\hline in4 & 144 & 532 & 2150080 & $100.00 \%$ & $98.31 \%$ \\
\hline in5 & 45 & 228 & 591 & $100.00 \%$ & $45.61 \%$ \\
\hline rckl & 19 & 102 & 33 & $100.00 \%$ & $19.61 \%$ \\
\hline vg2 & 43 & 198 & 3748 & $100.00 \%$ & $100.00 \%$ \\
\hline xidn & 15 & 78 & 74 & $100.00 \%$ & $6.41 \%$ \\
\hline x6dn & 91 & $(+1) 421$ & 24944 & $100.00 \%$ & $100.00 \%$ \\
\hline x7dn & 24 & 104 & 182 & $100.00 \%$ & $99.04 \%$ \\
\hline x9dn & 17 & 82 & 74 & $100.00 \%$ & $30.49 \%$ \\
\hline Ave. & & & & $100.00 \%$ & $63.80 \%$ \\
\hline
\end{tabular}

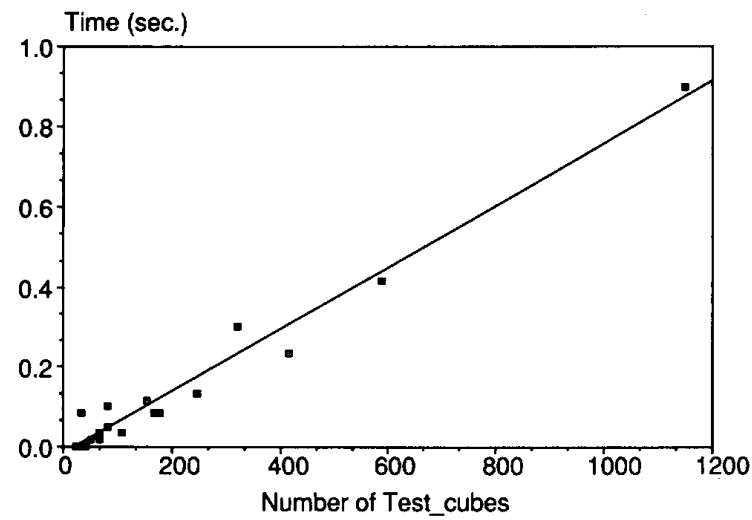

Fig. 7. The plots of computation times with respect to the numbers of "test_cubes" of the 18 benchmark functions run by FUTS in Table I.

For the algorithm CUTS to find UTS, it is the number of the operation of "comparing two vertices to determine whether they dominate each other," which costs the computation time. The number of "comparing" operations is approximately proportional to the square of the number of vertices which need to be "compared." So, for CUTS, the time complexity is $O\left(2^{n}\right)-O\left(2^{2 n}\right)$ for an n-input function. Yet, for the algorithm FUTS, since no comparing operation is involved, the time complexity is approximately linear with respect to the number of "test_cubes". To demonstrate the relationship between CPU time spent by FUTS and the number of test_cubes, Fig. 7 is plotted for the 18 functions in Table $I$. It is seen that a linear curve is obtained.

In addition, since the size of UTS grows exponentially with the number of binate inputs, the number of "comparing"

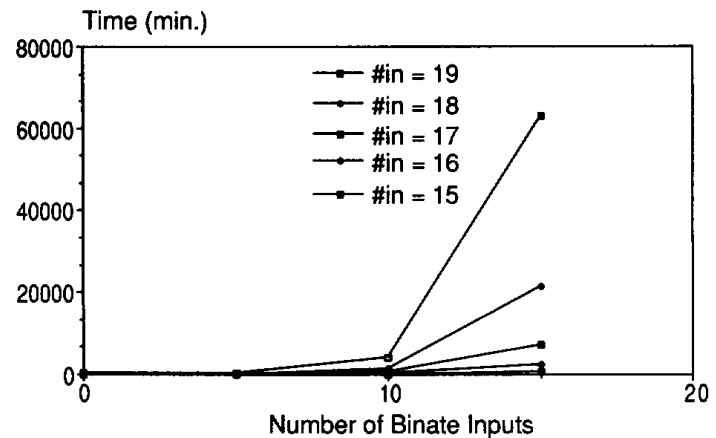

(a)

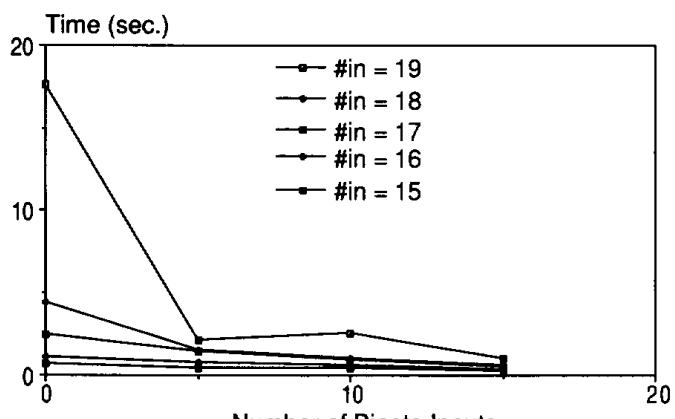

(b)

Fig. 8. The plots of computation times with respect to the numbers of binate inputs for the cases : input numbers $=15,16,17,18$, and 19 obtained by (a) CUTS and (b) FUTS respectively.

operations for CUTS to generate UTS increases rapidly with the number of binate inputs. For FUTS, on the contrary, the computation time decreases with the number of binate inputs since it Shannon-expands the function with respect to the binate input variables. This effectively applies a "divide-andconquer" strategy to solve the problem. To demonstrate this, CUTS and FUTS were applied to several functions, which were composed of randomly generated but equal numbers of product terms, to generate their UTS's. The computation times to generate UTS's are plotted in terms of the numbers of binate inputs in Fig. 8(a) and (b) for CUTS and FUTS respectively. In Fig. 8, the numbers of input variables for these functions were from 15 to 19 and the numbers of binate inputs were varied from 0 to 5, 10, and 15. In Fig. 8(a) and (b), two facts can be observed : First, the computation times for CUTS are much much larger than that of FUTS, and, second, the computation times for CUTS increase exponentially with the numbers of binate inputs while for FUTS the computation times decrease with the numbers of binate inputs.

\section{B. Results on MOUTS}

The procedure MOUTS has also been implemented in C language to run on a sun4/SPARC2 workstation. It was applied to run on the benchmark functions of [5]. The results are compiled in Table III. In the table, the numbers of inputs and outputs of each function are also listed. For comparison, the 
TABLE III

The Comparison of UTS Size and CPU Times for Test Generation Procedures With and Without tepact for 56 Benchmark Functions [5]

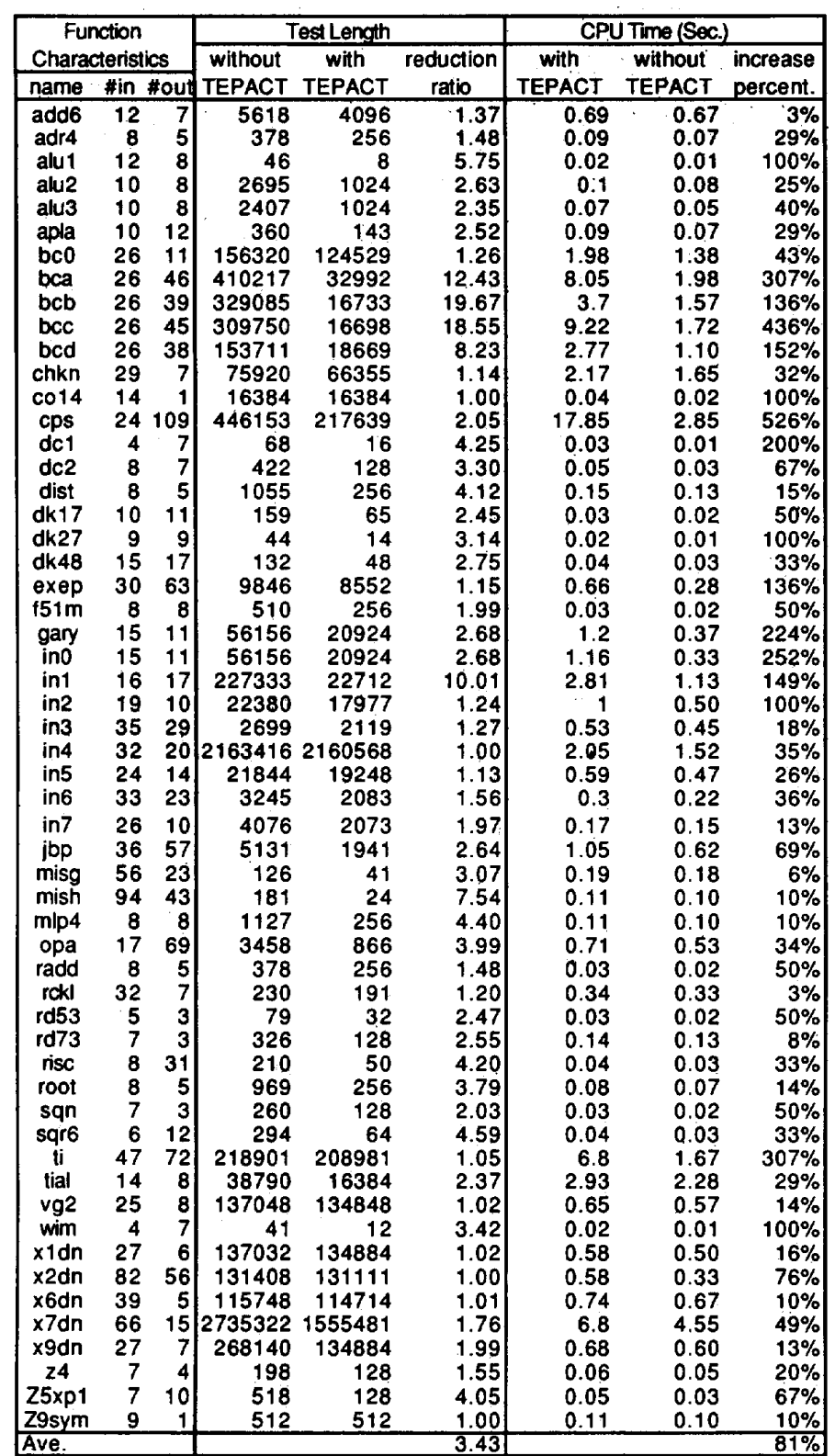

results run by the procedure which includes TEPACT and not TEPACT are listed. The fault coverages for each implemented circuit of the function covered by the UTSs generated by the procedure for two cases are all $100 \%$. It can be seen that the average reduction ratio on test lengths of UTSs generated with TEPACT to those generated without TEPACT is 3.4. For some functions, the reduction ratio is even as high as 20 . The increased CPU times to generate the UTS for each function with TEPACT is generally small. For these 56 functions, the average CPU time increase is $81 \%$. Also, it is seen that due to the fast algorithm FUTS, the CPU times are very small for each function.

\section{CONCluSion}

In this paper, a fast universal test set (UTS) generation for multi-output functions is presented.

First, this paper presents a fast algorithm to generate the UTS for single-output functions. The algorithm generates UTS from the algebraic representation directly to obtain test_cube set instead of enumerating the expanded truth table to obtain test pattern set. This significantly reduces the CPU time and the requirement of temporary memory and memory storage for tests. Experimental results show that the algorithm achieves an improvement of $10^{2}-10^{6}$ fold in the CPU time to compute 
UTS and a saving of $1-1800$ fold in memory storage to store UTS over the conventional method.

Then this paper presents a fast procedure to generate UTS for multi-output functions. It has been shown that the compaction technique used in the procedure achieved a reduction of 1-20 times in test lengths for the benchmark functions. For the most time-cost function: cps, which has 24 inputs and 109 outputs, only $18 \mathrm{~s}$ of sun4/SPARC2 CPU time is needed to obtain its UTS. From the experimental results, we could claim that the algorithms in this paper make the UTS generation practical for multi-output functions.

\section{REFERENCES}

[1] R. McNaughton, "Unate truth functions," IRE Trans. Electron. Comput., vol. EC-10, pp. 1-6, Mar. 1961

[2] S. B. Akers, "Universal test sets for logic networks," IEEE Trans. Computers, vol. C-22, pp. 835-839, Sept. 1973.

[3] S. M. Reddy, "Complete test sets for logic functions," IEEE Trans. Computers, vol. C-22, pp. 1016-1020, Nov. 1973.

[4] G. Gupta and N. K. Jha, "A universal test set for CMOS circuits," IEEE Trans. Computer-Aided Design, vol. CAD-7, pp. 590-597, May 1988.

[5] R. K. Brayton, G. D. Hachtel, C. T. McMullen, and A. L. SangiovanniVincentelli, Logic Minimization Algorithms for VLSI Synthesis. Hingham, MA: Kluwer Academic, 1984.

[6] S. J. Hong, R. G. Cain, and D. L. Ostapko, "MINI: A heuristic approach for logic minimization," IBM J. Res. Develop., vol. 18, pp. 443-458, Sept. 1974
[7] R. K. Brayton, R. L. Rudell, A. Sangiovanni-Vincentelli, and A. R. Wang, "MIS: A multilevel logic optimization system," IEEE Trans. Computer-Aided Design, vol. CAD-6, pp. 1062-1081, Nov. 1987.

Beyin Chen received the B. S. degree in electronics engineering in 1987 from the National Chiao Tung University, Taiwan, Republic of China, where presently she is working towards the $\mathrm{Ph}$. D. degree.

Her research interests include VLSI testing and logic verification.

Chung Len Lee (S'70-M'75-SM'92) received the B. S. degree from National Taiwan University, Taiwan, Republic of China, and the M. S. and Ph. D. degrees from Camegie-Mellon University, Pittsburgh, PA, all in electrical engineering, in 1968, 1971, and 1975 , respectively.

He joined the Department of Electronics Engineering, National Chiao Tung University in 1975, where presently he is a Professor. His teaching and research have been in the areas of integrated circuits and testing. $\mathrm{He}$ has supervised more than $90 \mathrm{M}$. S. and $\mathrm{Ph}$. D. students to complete their thesis and has published more than 140 papers in technical journals and conferences in the above areas.

Presently, Dr. Lee is a member of the editorial board of Journal of Electronic Testing, Theory and Application (Kluwer) and the lEEE Asian Test Technology Committee. 